\title{
In vivo treatment of rat arterial adventitia with interleukin-1 $\beta$ induces intimal proliferation via the JAK2/STAT3 signaling pathway
}

\author{
XIAO WANG ${ }^{1 *}$, LIHUA CHEN ${ }^{2 *}$, JIE LIU $^{1}$, TAO YAN $^{3}$, GANGYONG WU $^{1}$, \\ YANG XIA $^{1}$, GANGJUN ZONG ${ }^{1}$ and FENGSHENG $\mathrm{LI}^{3}$ \\ Departments of ${ }^{1}$ Cardiovascular Diseases and ${ }^{2}$ Radiology, The 101st Hospital of PLA, Wuxi, Jiangsu 214044; \\ ${ }^{3}$ Central Laboratories, The Second Artillery General Hospital, Beijing 100088, P.R. China
}

Received April 4, 2015; Accepted February 9, 2016

DOI: $10.3892 / \mathrm{mmr} .2016 .4982$

\begin{abstract}
Previous studies have indicated that adventitial inflammation is involved in the development of atherosclerosis. The aim of this study was to investigate the effect of arterial adventitia inflammation induced by interleukin (IL)- $1 \beta$ on intimal proliferation and the mechanisms involved in this process. The left common carotid artery adventitia of male rats in the experimental and control groups (25 rats/group) was wrapped with agar containing or without a sustained-release suspension of $2.5 \mu \mathrm{g}$ IL-1 $\beta$, respectively. Five animals in each group were randomly selected for sacrifice at $2 \mathrm{~h}, 8 \mathrm{~h}, 24 \mathrm{~h}, 48 \mathrm{~h}$, and 1 week post-treatment. Hematoxylin and eosin staining was performed for to analyze the morphology of the adventitia. The expression of janus kinase (JAK)2, signal transducer and activator of transcription (STAT)3, phosphorylated (p-)JAK2 and p-STAT3 were detected by western blot analysis or immunohistochemistry staining. A model of adventitial inflammation was successfully created by wrapping IL-1 $\beta$ around the rat carotid artery. IL-1 $\beta$ treatment induced vascular smooth muscle cell proliferation and migration as well as intimal proliferation. In addition, the expression of p-JAK2 and p-STAT3 increased after IL-1 $\beta$ treatment. Furthermore, an inhibitor of JAK2/STAT3 pathway, AG490, suppressed
\end{abstract}

Correspondence to: Dr Gangjun Zong, Department of Cardiovascular Diseases, The 101st Hospital of PLA, 3 Xingyuanbei Street, Wuxi, Jiangsu 214044, P.R. China

E-mail: 13366239527@163.com

Dr Fengsheng Li, Central Laboratories, The Second Artillery General Hospital, 16 Xinwai Street, Beijing 100088, P.R. China

E-mail: lifs0624@163.com

*Contributed equally

Key words: adventitial inflammation, intimal proliferation, janus kinase 2/signal transducer and activator of transcription 3 signaling pathway
IL-1 $\beta$-induced intimal proliferation and phosphorylation of JAK2 and STAT3. Thus, the JAK2/STAT3 signaling pathway is involved in intimal proliferation caused by vascular adventitial inflammation. Inhibiting the JAK2/STAT3 signaling pathway may be a novel method for the clinical treatment of artery atherosclerosis.

\section{Introduction}

Atherosclerosis is a complicated vascular disease, and is generally recognized as a chronic inflammatory disease (1-3). It is characterized by infiltration of various inflammatory cells, including $\mathrm{T}$ cells, mast cells and macrophages, into atherosclerotic plaques at all stages of development of atherosclerosis (4). Atherosclerosis is initiated by subendothelial retention of apolipoprotein B-containing lipoproteins followed by adhesion and penetration of inflammatory cells from the blood into the arterial intima (5). By secreting cytokines and growth factors, these inflammatory cells, together with resident vascular wall cells, can promote the migration and proliferation of vascular smooth muscle cells (VSMCs), which participate in the formation of neointima and finally plaque formation (6-8).

In addition to intimal inflammation, numerous previous studies have focused on the correlation between adventitial inflammation and atherosclerosis. Schwartz and Mitchell (9) were the first to report that cellular infiltration of the adventitia is associated with atheromatous plaques in 1962. T lymphocytes and B lymphocytes, together with a small proportion of macrophages, have been observed in areas of adventitial inflammation $(10,11)$. Furthermore, a previous study demonstrated that the degree of adventitial inflammation is positively correlated with the severity of atherosclerotic lesion (11). Although a number of studies support the concept that adventitial inflammation is derived from the spread of intimal inflammation (12-14), a recent study showed that adventitial inflammation may be responsible for the accumulation of macrophages in the intima and involved in atherosclerotic lesion development as an important early event $(6,15,16)$. Shimokawa et al $(17)$ suggested that in vivo chronic treatment of the coronary adventitia with interleukin 
(IL)-1 $\beta$ can induce intimal thickening, which is the earliest pathological change in atherosclerosis. Subsequently, the authors further found that treatment of the coronary adventitia with other key inflammatory cytokines [tumor necrosis factor (TNF)- $\alpha$ and IL-1 $\alpha$ ] also induces arteriosclerosis-like changes in the coronary intima (18). These studies strongly indicate that adventitial inflammation can trigger the development of atherosclerosis; however, the mechanism involved in this process has not been clearly defined.

As mentioned, inflammation-induced VSMC proliferation and migration from the media to intima are key early events in lesion development (19). VSMCs respond to specific extracellular stimuli secreted by inflammatory cells or vascular wall cells to induce signal transduction to the nucleus. This activates the expression of a series of genes controlling VSMC proliferation and migration, thus promoting VSMC migration from the media to the intima as well as VSMC proliferation. Previous studies have shown that the Janus kinase/signal transducer and activator of transcription (JAK/STAT) signaling pathway is important role in atherosclerotic lesion development (20-22). Notably, it has been verified that the JAK2/STAT3 signaling pathway is critical in VSMC proliferation and migration (23-25). Tyrosine phosphorylation occurs sequentially on JAK2 and STAT3, which in turn activates downstream target gene expression influencing VSMC proliferation and migration (26-30). In the current study, the adventitial inflammatory process was initiated by an wrapping agar suspension containing interleukin (IL)- $1 \beta$ around the adventitia to determine the role of the JAK2/STAT3 signaling pathway in mediating adventitial inflammation-induced vascular proliferation and lesion formation.

\section{Materials and methods}

Animals. A total of 15, ten-week old, male Sprague-Dawley (SD) rats (weight, 180-200 g) were obtained from the Vital River Laboratories, Co., Ltd. (Beijing, China). All rats were housed at $22^{\circ} \mathrm{C}$ with a $12: 12$-h light-dark cycle and free access to standard rodent chow and tap water in a rodent facility. Animal experiments were approved by the Animal Research Committee of the 101st Hospital of PLA (Wuxi, China). All procedures were performed in accordance with the guidelines of the Animal Research Committee of the 101st Hospital.

Preparation of $I L-1 \beta$ sustained-release agar suspension. A 100-mg slurry of CNBr-activated Sepharose 4B (45-165 $\mu \mathrm{m}$ diameter; Sigma-Aldrich, St. Louis, MO, USA) was washed with $1 \mathrm{mM} \mathrm{HCl}$ by vortexing and centrifugation $(328 \mathrm{x} \mathrm{g}$ for $5 \mathrm{~min}$ ) four times. The slurry was then washed three times with a solution containing $0.5 \mathrm{M} \mathrm{NaCl}$ and $1 \mathrm{M} \mathrm{NaHCO}_{3}$, followed by centrifugation ( $328 \mathrm{x}$ g for $5 \mathrm{~min}$ ). Then, $100 \mu \mathrm{g}$ IL-1 $\beta$ (Beijing Sino Biological Technology Co., Ltd., Beijing, China) was added to the slurry precipitation and mixed thoroughly. This mixture was incubated at room temperature for $1 \mathrm{~h}$ followed by incubation at $4^{\circ} \mathrm{C}$ overnight. The following day, the sample was centrifuged briefly for $5 \mathrm{~min}$ at $328 \mathrm{xg}$ and the liquid was discarded. The remaining solid phase was suspended in $1 \mathrm{M}$ Tris- $\mathrm{HCl}$ to form a slurry, and the mixture was incubated at room temperature for $1 \mathrm{~h}$, it was then centrifuged and the liquid was removed. Saline was added and the beads were washed 3 times and resuspended in $2 \mathrm{ml}$ saline. The final concentration of IL- $1 \beta$ was $50 \mathrm{mg} / \mathrm{ml}$.

Tyrosine kinase (JAK2) inhibitor AG490 sustained-release gel preparation. P-F127 (0.3 g; Sigma-Aldrich) was dissolved in $1 \mathrm{ml}$ phosphate-buffered saline (PBS), and then $30 \mu \mathrm{g}$ AG490 (Sigma-Aldrich) was added. This mixture was vortexed and incubated at $4^{\circ} \mathrm{C}$ overnight. The final concentration of AG490 was $100 \mu \mathrm{M}$.

Construction of the adventitial inflammation-induced intimal proliferation rat model. Fifty SD male rats were randomly divided into an experimental group and a control group $(n=25)$. Prior to surgery, rats were anesthetized by intraperitoneal injection of $2 \%$ sodium pentobarbital ( $50 \mathrm{mg} / \mathrm{kg}$ body weight; Sigma-Aldrich). The animals were supine-fixed on a surgical board. Following removing the neck hair using a razor and routinely disinfecting the skin using iodophor disinfection solution, a $3-\mathrm{cm}$ longitudinal incision was made along the midline to expose 2 to $3 \mathrm{~cm}$ of the left common carotid artery. Gauze $(2 \times 0.5 \mathrm{~cm}$; Medicom Inc., Ltd., Shanghai, China) was placed beneath the artery. For the experimental group, $50 \mu 1$ agar suspensions with IL- $1 \beta$ was placed on the gauze, and for the control group, $50 \mu \mathrm{l}$ of the agar suspension without IL-1 $\beta$ was placed on the gauze. The gauze was wrapped around the blood vessel using surgical cord (Medicom Inc., Ltd.) and sutures were used to close the incision. Sodium penicillin (100 kIU; Shandong Lukang Pharmaceutical Co., Ltd., Jining, China) was injected intramuscularly once every three days to prevent infection. The animals were given free access to standard rodent chow and tap water, and five animals from each group were sacrificed at $2 \mathrm{~h}, 8 \mathrm{~h}, 24 \mathrm{~h}, 48 \mathrm{~h}$ and 1 week post-surgery. Target blood vessels were excised $(\sim 4 \mathrm{~mm})$ and fixed in $4 \%$ paraformaldehyde (Beyotime Institute of Biotechnology, Shanghai, China) and the remaining tissue was frozen in liquid nitrogen. For AG490 treatment, both sides of the carotid artery of SD male rats $(n=15)$ were surgically separated, and an experimental side and a control side were assigned. For experimental sides, $150 \mu 1 \mathrm{AG} 490$ sustained release P-F127 gel was applied, and for control sides, $150 \mu 1$ gel with blank solution was applied. Subsequently, $50 \mu 1$ agar suspension with IL-1 $\beta$ was applied to both sides, and surgery was completed as described above. Five animals were randomly selected for sacrifice at $8 \mathrm{~h}$, $48 \mathrm{~h}$, and 1 week post-surgery, and samples were obtained as described above.

Histopathological examination. Conventional methods were used to fix and dehydrate the vascular tissue, and embed the tissues in paraffin (Chuandong Chemical Co., Ltd., Chongqing, China). The paraffin-embedded tissues were cut into 4- $\mu \mathrm{m}$ slices, and sections were stained using hematoxylin and eosin (H\&E; Beyotime Institute of Biotechnology). Changes in blood vessel layers were observed using a microscope (BX51; Olympus, Tokyo, Japan). Image-Pro Plus 6.0 software (Media Cybernetics, Rockville, MD, USA) was used to measure layer areas and calculate the intima/media ratio (IMR). 
Western blot analysis. Proteins were extracted from the frozen vascular tissues by homogenization using E.Z.N.A DNA/RNA/Protein Isolation kit (Omega Bio-Tek, Inc., Norcross, GA, USA) and quantified using the bicinchoninic acid assay (Beyotime Institute of Biotechnology). In total, $15 \mu \mathrm{g}$ (to detect total JAK2 and STAT3 proteins) or $30 \mu \mathrm{g}$ (to detect phosphorylated proteins) protein were resolved using $8 \%$ sodium dodecyl sulfate-polyacrylamide gel electrophoresis and transferred to polyvinylidene fluoride membranes (Merck Millipore, Billerica, MA, USA). Membranes were blocked with $5 \%$ bovine serum albumin (Sigma-Aldrich) for $1 \mathrm{~h}$ at room temperature, and then incubated with rabbit polyclonal anti-rat STAT3 antibody (1:400; cat. no. sc-7179; Santa Cruz Biotechnology, Inc., Santa Cruz, CA, USA), rabbit polyclonal anti-rat JAK2 antibody (1:1,000, cat. no. ab39636; Epitomics, Burlingame, CA, USA), rabbit polyclonal anti-rat phosphorylated JAK2 (p-JAK2) antibody (1:1,000; cat. no. ab68268; Epitomics), and rabbit anti-rat phosphorylated STAT3 (p-STAT3) antibodies (1:1,000; cat. no. ab76315; Epitomics) overnight at $4^{\circ} \mathrm{C}$ overnight. Rabbit anti-glyceraldehyde-3-phosphate dehydrogenase antibody (1:1,000; cat. no. sc-25778; Santa Cruz Biotechnology, Inc.) was used as a loading control for normalization. Membranes were washed and incubated with goat anti-rabbit horseradish peroxidase-conjugated secondary antibody (1:5,000; cat. no. zb-2301; Beijing Zhongshan Jinqiao Biotechnology Co., Ltd., Beijing, China) at $37^{\circ} \mathrm{C}$ for $1 \mathrm{~h}$. Chemiluminescence was detected using an enhanced chemiluminescence reagent, and Quantity One software (version 4.6.2; Bio-Rad Laboratories, Inc., Hercules, CA, USA) was used to measure band intensity.

Immunohistochemical staining. Deparaffinized and rehydrated tissue sections were placed in a microwavable vessel with antigen retrieval buffer (citric acid-sodium citrate buffer, $\mathrm{pH}$ 6.0) and exposed to microwaves for $15 \mathrm{~min}$ in a microwave oven at $750 \mathrm{~W}$. Using an Envision immunohistochemical staining kit (Dako, Carpinteria, CA, USA), paraffin sections were subsequently stained with rabbit anti-rat $\alpha$-actin polyclonal antibody (1:100; cat. no. sc-53142; Santa Cruz Biotechnology, Inc.), rabbit polyclonal anti-rat proliferating cell nuclear antigen (PCNA; 1:100; cat. no. sc-7907; Santa Cruz Biotechnology, Inc.), rabbit polyclonal anti-rat embryonic smooth muscle myosin heavy chain (SM-emb) antibody (1:100; cat. no. sc-65734; Santa Cruz Biotechnology, Inc.), rabbit polyclonal anti-rat p-JAK2 (1:100; cat. no. kg11151; Nanjing KeyGen Biotech. Co., Ltd., Nanjing, China), rabbit polyclonal anti-rat p-STAT3 (1:100; cat. no. kg11045-1; Nanjing KeyGen Biotech. Co., Ltd.) to determine the corresponding proteins in tissues.

Statistical analysis. SPSS17.0 software (SPSS Software, Inc., Chicago, IL, USA) was used to perform statistical analysis. Experimental data were collected from multiple animals under each experimental condition $(n=5)$ and are presented as the mean \pm standard deviation. One-way analysis of variance was conducted to compare means of three or more groups. The Student-Newman-Keuls (SNK) method was used for pairwise comparisons. A t-test was used to compare vascular protein intensity from the left and right sides of the same rat. $\mathrm{P}<0.05$, was considered to indicate a statistically significant difference.
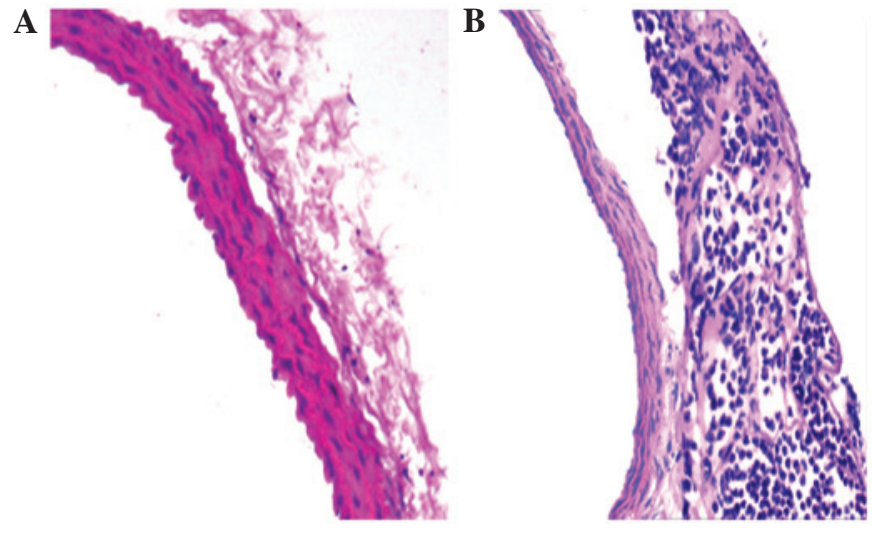

Figure 1. Adventitial inflammation was triggered by treatment with interleukin-1 $\beta$. Vascular tissue pathology of a rat from (A) the control group and (B) the experimental group $2 \mathrm{~h}$ post-surgery was examined by light microscopy and hematoxylin and eosin staining (magnification, $\mathrm{x} 400$ ).

\section{Results}

Adventitial administration of $I L-1 \beta$ induces smooth muscle cell proliferation, migration, and neointimal formation. The model of adventitial inflammation induced by treatment with IL-1 $\beta$ has been well documented in previous study (17). In the present study, the results of H\&E staining demonstrated leucocyte, including numerous neutrophils, infiltration into the vascular adventitia $2 \mathrm{~h}$ after surgery (Fig. 1), which indicated that adventitial inflammation was triggered and the model was successfully established.

At all the time points, the vascular morphology of the control group was normal and no intimal proliferation responses were observed (Fig. 2A). After surgery (2 h), cell proliferation and migration towards the lumen were visible in the experimental group (Fig. 2B). At $8 \mathrm{~h}$, intimal proliferation and luminal narrowing of the blood vessels was observed, which was shown by H\&E staining (Fig. 2C) and immunohistochemical staining with anti-rat PCNA antibody (Fig. 2D), respectively. Further immunohistochemical staining with the anti-rat $\alpha$-actin antibody (Fig. 2E) and anti-rat SM-emb antibody (Fig. 2F) showed positive staining in the majority of neointimal cells, indicating that the cells that has proliferated and migrated towards the lumen were VSMCs. Statistical analysis of IMR results from different time points revealed significant differences in intima proliferation over time $(\mathrm{P}<0.05)$. As early as $8 \mathrm{~h}$ post-surgery, the IMR of mice in the experimental group was significantly higher than that in the control group $(\mathrm{P}<0.05$; Fig. $2 \mathrm{G})$.

Adventitial administration of IL-1 $\beta$ activates the JAK2/STAT3 pathway in vascular tissue. After the adventitial inflammatory response was induced by treatment with IL-1 $\beta$, protein expression of members of the JAK2/STAT3 pathway were evaluated using western blotting. As shown in Fig. 3A and B, the vascular expression of total JAK2 and STAT3 was comparable between the experimental group and the control group at all the examined time points. However, treatment with IL-1 $\beta$ resulted in a gradual and progressive increase in vascular p-JAK 2 which peaked at $8 \mathrm{~h}$ post-treatment, and then gradually decreased to normal levels. Similarly, after IL-1 $\beta$ treatment, the p-STAT3 level increased at $2 \mathrm{~h}$ post-treatment, peaked at 

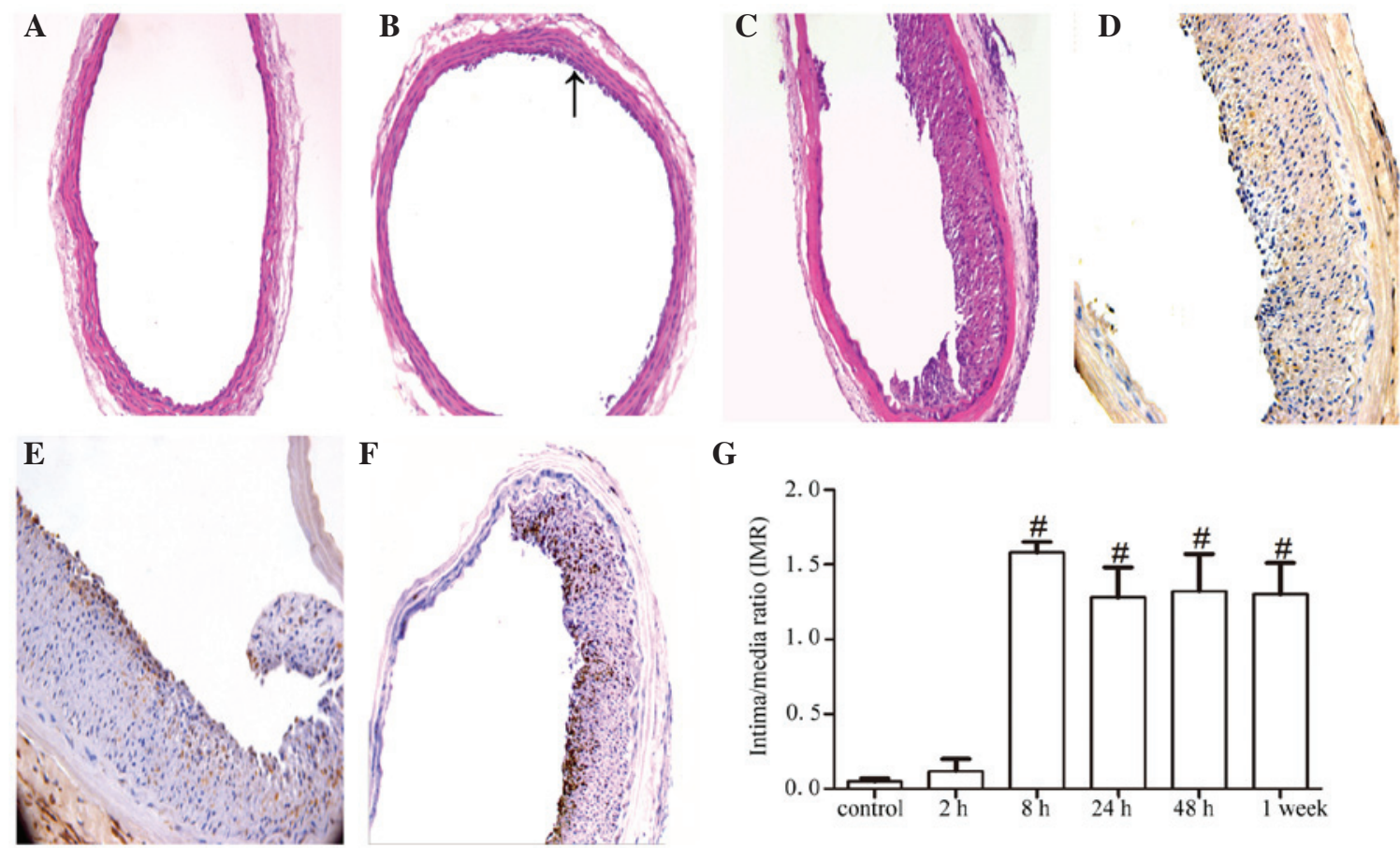

G

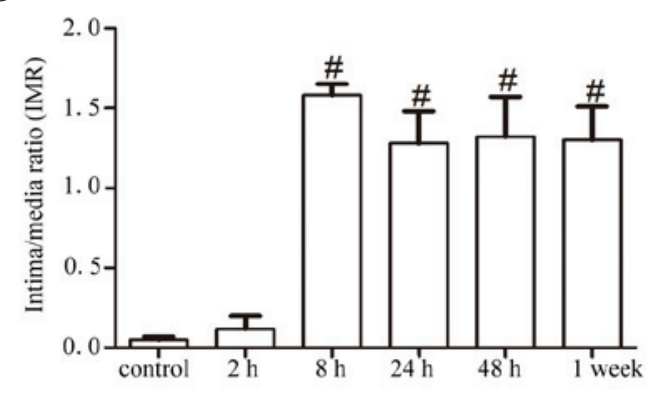

Figure 2. Adventitial administration of interleukin-1 $\beta$ induced smooth muscle cell proliferation, migration and neointima formation. Vascular tissue pathology of rats from (A) the control group, and the experimental group (B) $2 \mathrm{~h}$ and (C) $8 \mathrm{~h}$ post-surgery was examined by light microscopy and hematoxylin and eosin staining (magnification, $\mathrm{x} 200$ ). Arrows in B indicate cell proliferation and migration. Immunohistochemical staining was performed to detect the expression of (D) proliferating cell nuclear antigen, (E) $\alpha$-actin and (F) embryonic smooth muscle myosin heavy chain in the vascular tissue derived from the experimental group $8 \mathrm{~h}$ post-surgery (magnification, $\mathrm{x} 400$ ). (G) Statistical analysis of the intima/media ratio. The results are expressed as the mean \pm standard deviation $(\mathrm{n}=5) .{ }^{*} \mathrm{P}<0.05$ vs. control.

A control $2 \mathrm{~h} \quad 8 \mathrm{~h} \quad 24 \mathrm{~h} \quad 48 \mathrm{~h} \quad 1$ week

B
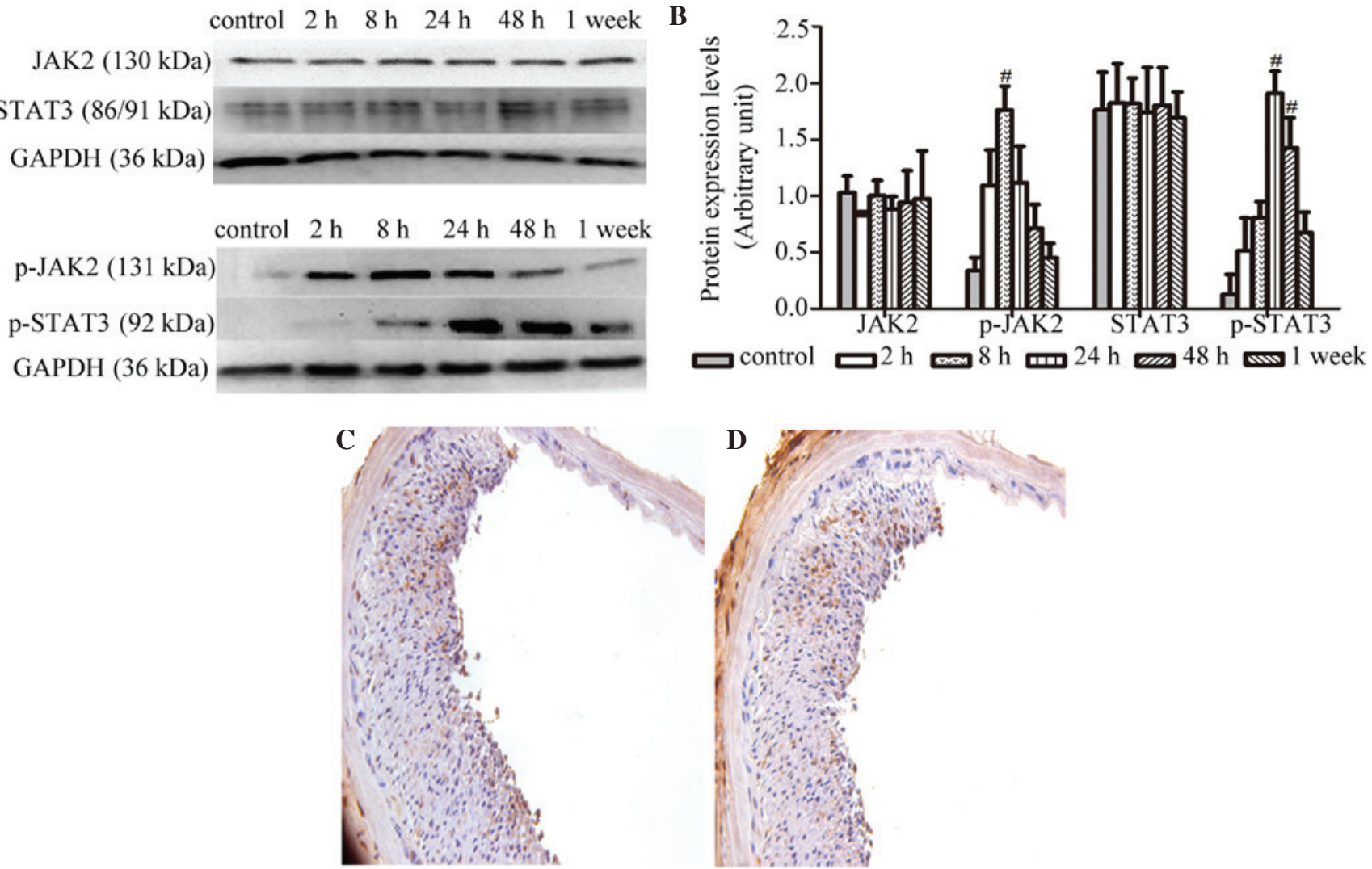

Figure 3. Adventitial administration of interleukin-1 $\beta$ activated the JAK2/STAT3 pathway in the vascular tissue. (A) Protein expression levels of JAK2, STAT3, p-JAK2 and p-STAT3 in vascular tissue analyzed by western blot analysis. (B) Gray scale analysis of JAK2, STAT3, p-JAK2 and p-STAT3 protein expression levels. The results are expressed as the mean \pm standard deviation $(\mathrm{n}=5) .{ }^{*} \mathrm{P}<0.05$ vs. the control. Immunohistochemical staining was conducted to detect the expression of (C) p-JAK2 and (D) p-STAT3 in the vascular tissue derived from experimental group $8 \mathrm{~h}$ post-surgery (magnification, $\mathrm{x} 400$ ). JAK, janus kinase; STAT, signal transducer and activator of transcription; p-, phosphorylated; GAPDH, glyceraldehyde 3-phosphate dehydrogenase. 


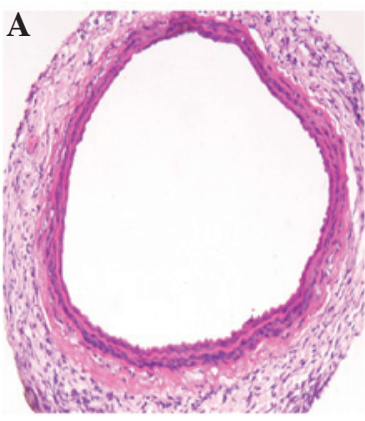

D

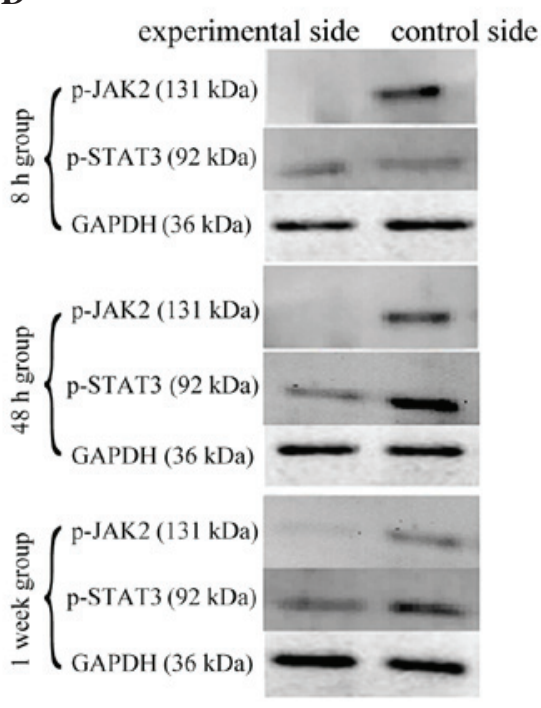

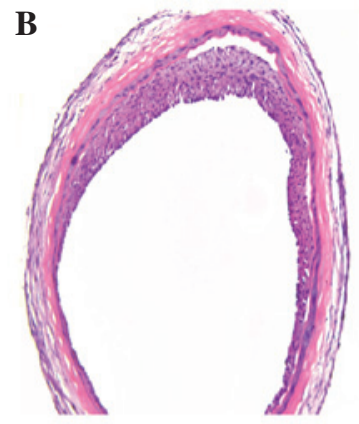

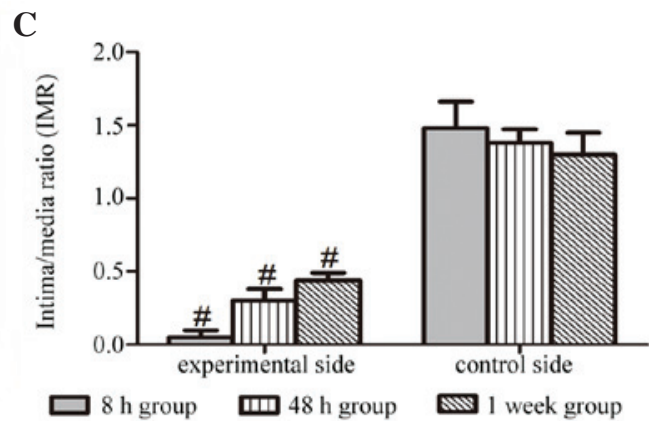

E

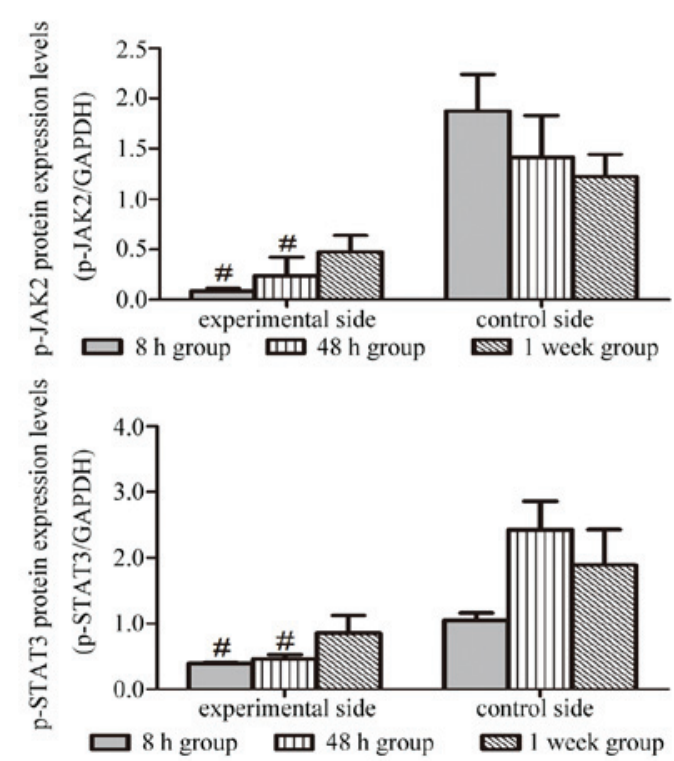

Figure 4. AG490 inhibits intimal proliferation, and p-JAK2 and p-STAT3 protein expression. After surgery ( $8 \mathrm{~h}$ ), vascular tissue pathology of (A) experimental side (treated with AG490 and IL-1 $\beta$ ) and (B) control side (treated with IL-1 $\beta$ only) tissue was examined by light microscopy and hematoxylin and eosin staining (magnification, x200). (C) Statistical analysis of the intima/media ratio. (D) Protein expression levels of p-JAK2 and p-STAT3 in vascular tissue by western blot analysis. (E) Gray scale analysis of p-JAK2, and p-STAT3 protein expression levels. The results are expressed as the mean \pm SD $(n=5)$. ${ }^{*} \mathrm{P}<0.05$ vs. the control side. IL, interleukin; p-, phosphorylated; JAK, janus kinase; STAT, signal transducer and activator of transcription; GAPDH, glyceraldehyde 3-phosphate dehydrogenase.

$24 \mathrm{~h}$ post-treatment, was maintained until $48 \mathrm{~h}$ post-treatment, and then gradually decreased. Immunohistochemical staining revealed that p-JAK2 and p-STAT3 were expressed in VSMCs, particularly in proliferating cells and migrating cells within the lumen and near the neointima (Fig. 3C and D).

AG490 administration inhibits IL-1 $\beta$-induced intimal proliferation and phosphorylation of JAK2 and STAT3. Compared with the control group, VSMC proliferation and migration, and intimal proliferation in the experimental group administered the JAK2 inhibitor (AG490) were significantly suppressed (Fig. 4A and B). IMR measurements and statistical analysis showed that the arterial IMR of rats in the AG490-treated group was significantly decreased compared that in the group without AG490 treatment at all the examined time points (Fig. 4C).

The effect of AG490 administration on the IL-1 $\beta$-increased expression of p-JAK2 and p-STAT3 was also investigated by western blot analysis. As shown in Fig. 4D and E, The IL-1 $\beta$-increased expression of p-JAK2 was significantly inhibited by AG490 administration at the 8 and $48 \mathrm{~h}$ time points. One week after treatment, although the expression of p-JAK2 in the artery of experimental side was lower than that of control side, there was no significant difference between the two values. Similarly, the increase of p-STAT3 induced by IL-1 $\beta$ was also suppressed by AG490 treatment at 8 and $48 \mathrm{~h}$ time points, and no significant difference in the expression of p-STAT3 was observed between the two groups at 1 week.

\section{Discussion}

Studies have demonstrated that artery adventitial inflammation is involved in the initiation and development of atherosclerosis. Chemical cues, such as endotoxin, lipopolysaccharide and interleukins, have been used to induce adventitial inflammation (31). In the present study, an adventitial inflammation model was successfully created by wrapping IL- $1 \beta$ around the rat carotid. In this model, adventitial inflammation induced VSMC proliferation and migration as well as intimal proliferation. In addition, the expression of p-JAK2 and p-STAT3 increased following IL-1 $\beta$ treatment. Furthermore, an inhibitor of JAK2/STAT3 pathway, AG490, suppressed IL-1 $\beta$-induced intimal proliferation and phosphorylation of JAK2 and STAT3.

Neointimal formation, which is considered to be a marker for early atherosclerosis, is the main pathological process of atherosclerosis $(32,33)$. It has been reported that the excessive 
proliferation and migration of VSMCs from the arterial media into the intima under the stimulation of cytokines and growth factors participates in neointimal formation during the development of atherosclerosis (6-8) or restenosis after percutaneous transluminal coronary angioplasty (34). In the current study, the results showed that adventitial treatment with IL-1 $\beta$ resulted in neointimal formation. Furthermore, immunohistochemical staining with antibody against rat $\alpha$-actin and SM-emb, markers of VSMCs, indicated that VSMCs had migrated into the neointima, and were the main cellular component of the neointima, confirming their role in neointima formation. These results suggested that the initial step in atherosclerosis had been triggered by adventitial inflammation induced by treatment with IL-1 $\beta$, which was consistent with the results of a previous study (17).

The JAK2/STAT3 pathway can be triggered when a growth factor or cytokine binds to its receptor which then phosphorylates JAK2. The phosphorylated JAK2 subsequently phosphorylates STAT3, which results in the dimerization and translocation of STAT3 to the nucleus. Finally, STAT3 in the nucleus binds to the promoter region of its target genes to regulate the biological behavior of cells (35). The JAK2/STAT3 pathway is involved in numerous critical functions in normal and malignant cells, such as differentiation, proliferation, survival and angiogenesis $(36,37)$. During the development of atherosclerosis, activation of the JAK/STAT pathway, including the JAK2/STAT3 pathway have been observed in atherosclerotic lesions $(38,39)$. Moreover, the JAK2/STAT3 pathway mediates the proliferation and migration of VSMCs (25). In the current study, expression of JAK2 and STAT3 was analyzed to determine the time course of induction by IL-1 $1 \beta$. Immunohistochemistry revealed that following stimulation, the phosphorylation of JAK2 and STAT3 occurred primarily in proliferating and migrating VSMCs, indicating that VSMCs migrate and proliferate following autophosphorylation rather than indirect activation via other cells. The results of western blot analysis demonstrated that following IL-1 $\beta$ administration, JAK2 phosphorylation occurred at $2 \mathrm{~h}$ and peaked at $8 \mathrm{~h}$; and STAT3 phosphorylation was initiated at $2 \mathrm{~h}$ and peaked at $24 \mathrm{~h}$, remaining elevated for up to a week. These results indicated that the JAK2/STAT3 pathway was activated at the early stages of IL- $1 \beta$ treatment. In a rat carotid artery balloon injury experiment, JAK2 and STAT3 tyrosine phosphorylation levels peaked after 1 week, and VSMC proliferation and migration appeared $96 \mathrm{~h}$ post-surgery (40). In the present study, neointimal changes were observed as early as $8 \mathrm{~h}$ post-surgery, and phosphorylation levels of the two proteins peaked relatively earlier, indicating that JAK2 and STAT3 phosphorylation levels are correlated with VSMC proliferation and migration.

Numerous studies have shown that AG490 is a JAK2 inhibitor, which competes for binding with tyrosine kinases, thus inhibiting JAK2 phosphorylation. In the present study, 8 and 48 h post-AG490 treatment, p-JAK ${ }_{2}$ levels were lower than the detection range of western blot analysis. Although minimal levels of $\mathrm{p}-\mathrm{JAK}_{2}$ could be detected 1 week post-AG490 treatment, this may have resulted from the reduction in AG490 effectiveness due to dose elimination by degradation. It can be inferred from this experiment that
AG490 administration inhibits the IL-1 $\beta$-induced activation of the JAK2/STAT3 pathway. Notably, IL-1 $\beta$-induced VSMC proliferation and migration and neointimal formation was also significantly suppressed following AG490 administration, which suggested that VSMC proliferation and migration, and neointimal formation induced by IL- $1 \beta$ is dependent on the JAK2/STAT3 signal transduction pathway. This result was similar to that of several previous studies that demonstrated that AG490 effectively inhibits VSMC proliferation and migration by inhibiting JAK2 phosphorylation (41-43). However, the downstream molecules of the JAK2/STAT3 signaling pathway regulating the final intimal proliferation process remain unclear. A previous study demonstrated that metalloproteinases (MMPs) regulate the migration, proliferation, and death of VSMCs (44). Furthermore, members of MMPs family have been reported to be regulated by STAT3 $(45,46)$. These results imply that MMPs may act downstream of the JAK2/STAT3 signaling pathway and therefore functionally regulate the final intimal proliferation process induced by IL-1 $\beta$. This hypothesis requires further investigation.

In this study, IL-1 $\beta$ administration to the vascular adventitia was used as a model of adventitial inflammation, rat carotid artery VSMC proliferation, migration, and neointima formation were induced, which confirmed the role of adventitial inflammation in the development of atherosclerosis and was consistent with the results of a previous study (17), however the mechanisms underlying this remain unclear. The present study found that the JAK2/STAT3 signaling pathway was activated by adventitial inflammation, and administration of AG490 inhibited JAK2/STAT3 signaling accompanied by decreases in VSMC proliferation, migration, and neointima formation. These results indicate that the JAK2/STAT3 pathway is important in neointimal formation induced by adventitial inflammation and may be a potential therapeutic target for the clinical treatment of arterial atherosclerosis and restenosis following vascular injury. Notably, an oral JAK1/JAK2 inhibitor, ruxolitinib, has been approved to use in the treatment of myelofibrosis by the FDA (47), which indicates that agents blocking the JAK2/STAT3 signaling pathway are available in the clinic besides being feasible in theory.

Numerous major inflammatory cytokines have been demonstrated to induce arteriosclerosis-like changes in the coronary intima in various studies $(17,18)$. The current study, however, only investigated the mechanisms involved in IL-1 $\beta$-induced intimal proliferation. Further studies are required to explore whether the JAK2/STAT3 signaling pathway universally mediates intimal proliferation induced by these inflammatory cytokines.

\section{References}

1. Clarke MC, Talib S, Figg NL and Bennett MR: Vascular smooth muscle cell apoptosis induces interleukin-1-directed inflammation: Effects of hyperlipidemia-mediated inhibition of phagocytosis. Circ Res 106: 363-372, 2010.

2. Kleemann R, Zadelaar S and Kooistra T: Cytokines and atherosclerosis: A comprehensive review of studies in mice. Cardiovasc Res 79: 360-376, 2008.

3. Sprague AH and Khalil RA: Inflammatory cytokines in vascular dysfunction and vascular disease. Biochem Pharmacol 78: 539-552, 2009. 
4. Soehnlein O and Weber C: Myeloid cells in atherosclerosis: Initiators and decision shapers. Semin Immunopathol 31: 35-47, 2009.

5. Subbotin VM: Neovascularization of coronary tunica intima (DIT) is the cause of coronary atherosclerosis. Lipoproteins invade coronary intima via neovascularization from adventitial vasa vasorum, but not from the arterial lumen: A hypothesis. Theor Biol Med Model 9: 11, 2012.

6. Libby P, Ridker PM and Maseri A: Inflammation and atherosclerosis. Circulation 105: 1135-1143, 2002.

7. Lim S and Park S: Role of vascular smooth muscle cell in the inflammation of atherosclerosis. BMB Rep 47: 1-7, 2014.

8. Vogt F, Zernecke A, Beckner M, Krott N, Bosserhoff AK, Hoffmann R, Zandvoort MA, Jahnke T, Kelm M, Weber C and Blindt R: Blockade of angio-associated migratory cell protein inhibits smooth muscle cell migration and neointima formation in accelerated atherosclerosis. J Am Coll Cardiol 52: 302-311, 2008.

9. Schwartz CJ and Mitchell JR: Cellular infiltration of the human arterial adventitia associated with atheromatous plaques. Circulation 26: 73-78, 1962.

10. Watanabe M, Sangawa A, Sasaki Y, Yamashita M, Tanaka-Shintani M, Shintaku M and Ishikawa Y: Distribution of inflammatory cells in adventitia changed with advancing atherosclerosis of human coronary artery. J Atheroscler Thromb 14: 325-331, 2007.

11. Parums D and Mitchinson MJ: Demonstration of immunoglobulin in the neighbourhood of advanced atherosclerotic plaques. Atherosclerosis 38: 211-216, 1981

12. Zhao L, Moos MP, Gräbner R, Pédrono F, Fan J, Kaiser B John N, Schmidt S, Spanbroek R, Lötzer K, et al: The 5-lipoxygenase pathway promotes pathogenesis of hyperlipidemia-dependent aortic aneurysm. Nat Med 10: 966-973, 2004.

13. Moos MP, John N, Gräbner R, Nossmann S, Günther B, Vollandt R, Funk CD, Kaiser B and Habenicht AJ: The lamina adventitia is the major site of immune cell accumulation in standard chow-fed apolipoprotein E-deficient mice. Arterioscler Thromb Vasc Biol 25: 2386-2391, 2005.

14. Galkina E, Kadl A, Sanders J, Varughese D, Sarembock IJ and Ley K: Lymphocyte recruitment into the aortic wall before and during development of atherosclerosis is partially L-selectin dependent. J Exp Med 203: 1273-1282, 2006.

15. Rayner K, Van Eersel S, Groot PH and Reape TJ: Localisation of mRNA for JE/MCP-1 and its receptor CCR2 in atherosclerotic lesions of the ApoE knockout mouse. J Vasc Res 37: 93-102, 2000.

16. Kortelainen ML and Porvari K: Adventitial macrophage and lymphocyte accumulation accompanying early stages of human coronary atherogenesis. Cardiovasc Pathol 23: 193-197, 2014.

17. Shimokawa H, Ito A, Fukumoto Y, Kadokami T, Nakaike R, Sakata M, Takayanagi T, Egashira K and Takeshita A: Chronic treatment with interleukin-1 beta induces coronary intimal lesions and vasospastic responses in pigs in vivo. The role of platelet-derived growth factor. J Clin Invest 97: 769-776, 1996.

18. Fukumoto Y, Shimokawa H, Ito A, Kadokami T, Yonemitsu Y, Aikawa M, Owada MK, Egashira K, Sueishi K, Nagai R, et al: Inflammatory cytokines cause coronary arteriosclerosis-like changes and alterations in the smooth-muscle phenotypes in pigs. J Cardiovasc Pharmacol 29: 222-231, 1997.

19. Tedgui A and Mallat Z: Cytokines in atherosclerosis: Pathogenic and regulatory pathways. Physiol Rev 86: 515-581, 2006.

20. Liao M, Xu J, Clair AJ, Ehrman B, Graham LM and Eagleton MJ: Local and systemic alterations in signal transducers and activators of transcription (STAT) associated with human abdominal aortic aneurysms. J Surg Res 176: 321-328, 2012.

21. Mo ZC, Xiao J, Liu XH, Hu YW, Li XX, Yi GH, Wang Z, Tang YL, Liao DF and Tang CK: AOPPs inhibits cholesterol efflux by down-regulating ABCA1 expression in a JAK/STAT signaling pathway-dependent manner. J Atheroscler Thromb 18: 796-807, 2011.

22. Beyazit Y, Sayilir A, Suvak B and Torun S: The missing link between obesity and hepatocellular carcinoma: IL-6-mediated STAT-3 activation as a key player in hepatocarcinogenesis. Digestion 84: 185-186, 2011.

23. Manea A, Tanase LI, Raicu M and Simionescu M: Jak/STAT signaling pathway regulates nox 1 and nox4-based NADPH oxidase in human aortic smooth muscle cells. Arterioscler Thromb Vasc Biol 30: 105-112, 2010.
24. Ortiz-Munoz G, Martin-Ventura JL, Hernandez-Vargas P, Mallavia B, Lopez-Parra V, Lopez-Franco O, Muñoz-Garcia B, Fernandez-Vizarra P, Ortega L, Egido J and Gomez-Guerrero C: Suppressors of cytokine signaling modulate JAK/STAT-mediated cell responses during atherosclerosis. Arterioscler Thromb Vasc Biol 29: 525-531, 2009.

25. Song B, Jin H, Yu X, Zhang Z, Yu H, Ye J, Xu Y, Zhou T, Oudit GY, Ye JY, et al: Angiotensin-converting enzyme 2 attenuates oxidative stress and VSMC proliferation via the JAK2/STAT3/SOCS3 and profilin-1/MAPK signaling pathways. Regul Pept 185: 44-51, 2013.

26. Jinno T, Iwai M, Li Z, Li JM, Liu HW, Cui TX, Rakugi H, Ogihara T and Horiuchi M: Calcium channel blocker azelnidipine enhances vascular protective effects of AT1 receptor blocker olmesartan. Hypertension 43: 263-269, 2004

27. Banes AK, Shaw SM, Tawfik A, Patel BP, Ogbi S, Fulton D and Marrero MB: Activation of the JAK/STAT pathway in vascular smooth muscle by serotonin. Am J Physiol Cell Physiol 288: C805-C812, 2005

28. Sun J, Zheng J, Ling KH, Zhao K, Xie Z, Li B, Wang T, Zhu Z, Patel AN, Min W, et al: Preventing intimal thickening of vein grafts in vein artery bypass using STAT-3 siRNA. J Transl Med 10: 2, 2012

29. Dronadula N, Liu Z, Wang C, Cao H and Rao GN: STAT-3-dependent cytosolic phospholipase A2 expression is required for thrombin-induced vascular smooth muscle cell motility. J Biol Chem 280: 3112-3120, 2005.

30. Watanabe $\mathrm{S}, \mathrm{Mu} \mathrm{W}, \mathrm{Kahn} \mathrm{A}$, Jing $\mathrm{N}$, Li JH, Lan HY, Nakagawa T, Ohashi R and Johnson RJ: Role of JAK/STAT pathway in IL-6-induced activation of vascular smooth muscle cells. Am J Nephrol 24: 387-392, 2004.

31. Shimokawa H, Morishige K, Miyata K, Kandabashi T, Eto Y, Ikegaki I, Asano T, Kaibuchi $\mathrm{K}$ and Takeshita A: Long-term inhibition of Rho-kinase induces a regression of arteriosclerotic coronary lesions in a porcine model in vivo. Cardiovasc Res 51: 169-177, 2001.

32. Zeller I, Knoflach M, Seubert A, Kreutmayer SB, Stelzmüller ME, Wallnoefer E, Blunder S, Frotschnig S, Messner B, Willeit $\mathrm{J}$, et al: Lead contributes to arterial intimal hyperplasia through nuclear factor erythroid 2-related factor-mediated endothelial interleukin 8 synthesis and subsequent invasion of smooth muscle cells. Arterioscler Thromb Vasc Biol 30: 1733-1740, 2010.

33. Liu J, Sukhova GK, Sun JS, Xu WH, Libby P and Shi GP: Lysosomal cysteine proteases in atherosclerosis. Arterioscler Thromb Vasc Biol 24: 1359-1366, 2004

34. Pauletto P, Sartore S and Pessina AC: Smooth-muscle-cell proliferation and differentiation in neointima formation and vascular restenosis. Clin Sci (Lond) 87: 467-479, 1994.

35. Raible DJ, Frey LC and Brooks-Kayal AR: Effects of JAK2-STAT3 signaling after cerebral insults. JAKSTAT 3: e29510, 2014

36. Levy DE and Darnell JE Jr: Stats: Transcriptional control and biological impact. Nat Rev Mol Cell Biol 3: 651-662, 2002

37. Sansone $P$ and Bromberg J: Targeting the interleukin-6/Jak/stat pathway in human malignancies. J Clin Oncol 30: 1005-1014, 2012.

38. Seki Y, Kai H, Shibata R, Nagata T, Yasukawa H, Yoshimura A and Imaizumi T: Role of the JAK/STAT pathway in rat carotid artery remodeling after vascular injury. Circ Res 87: 12-18, 2000.

39. Recinos A III, LeJeune WS, Sun H, Lee CY, Tieu BC, Lu M, Hou T, Boldogh I, Tilton RG and Brasier AR: Angiotensin II induces IL-6 expression and the Jak-STAT3 pathway in aortic adventitia of LDL receptor-deficient mice. Atherosclerosis 194 125-133, 2007.

40. Shibata R, Kai H, Seki Y, Kato S, Wada Y, Hanakawa Y, Hashimoto K, Yoshimura A and Imaizumi T: Inhibition of STAT3 prevents neointima formation by inhibiting proliferation and promoting apoptosis of neointimal smooth muscle cells. Hum Gene Ther 14: 601-610, 2003.

41. Neria F, Caramelo C, Peinado H, González-Pacheco FR, Deudero JJ, de Solis AJ, Fernández-Sánchez R, Peñate $\mathrm{S}$, Cano A and Castilla MA: Mechanisms of endothelial cell protection by blockade of the JAK2 pathway. Am J Physiol Cell Physiol 292: C1123-C1131, 2007.

42. Neeli I, Liu Z, Dronadula N, Ma ZA and Rao GN: An essential role of the Jak-2/STAT-3/cytosolic phospholipase A(2) axis in platelet-derived growth factor BB-induced vascular smooth muscle cell motility. J Biol Chem 279: 46122-46128, 2004. 
43. Sasaguri T, Teruya H, Ishida A, Abumiya $\mathrm{T}$ and Ogata J: Linkage between alpha(1) adrenergic receptor and the Jak/STAT signaling pathway in vascular smooth muscle cells. Biochem Biophys Res Commun 268: 25-30, 2000.

44. Newby AC: Matrix metalloproteinases regulate migration, proliferation and death of vascular smooth muscle cells by degrading matrix and non-matrix substrates. Cardiovasc Res 69: 614-624, 2006.

45. Zhang L, Li Y, Liu Y, Wang X, Chen M, Xing Y and Zhu D: STAT3-mediated MMP-2 expression is required for 15-HETE-induced vascular adventitial fibroblast migration. J Steroid Biochem Mol Biol 149C: 106-117, 2015.
46. Jo DH, Kim JH, Cho CS, Cho YL, Jun HO, Yu YS, Min JK and Kim JH: STAT3 inhibition suppresses proliferation of retinoblastoma through down-regulation of positive feedback loop of STAT3/miR-17-92 clusters. Oncotarget 5: 11513-11525, 2014.

47. Mesa RA, Verstovsek S, Gupta V, Mascarenhas JO, Atallah E, Burn T, Sun W, Sandor V and Gotlib J: Effects of ruxolitinib treatment on metabolic and nutritional parameters in patients with myelofibrosis from COMFORT-I. Clin Lymphoma Myeloma Leuk 15: 214.e1-221.e1, 2015. 